\title{
1 Interregional causal influences of brain metabolic activity reveal the spread of aging effects during
}

2 normal aging

4 Xin Di ${ }^{1,2}$, Marie Wölfer ${ }^{1,3,4}$, Mario Amend ${ }^{5}$, Hans Wehrl ${ }^{5}$, Tudor M. Ionescu ${ }^{5}$, Bernd J. Pichler ${ }^{5}$, 5 Bharat B. Biswal ${ }^{1,2, *}$, the Alzheimer's Disease Neuroimaging Initiative ${ }^{\#}$

7 1. Department of Biomedical Engineering, New Jersey Institute of Technology, Newark, NJ, 07029, USA

8 2. School of Life Sciences and Technology, University of Electronic Science and Technology of China,

9 Chengdu, China

10 3. Clinical Affective Neuroimaging Laboratory (CANLAB), Otto-von-Guericke-University Magdeburg, 11 Magdeburg, Germany

12 4. Department for Behavioral Neurology, Leibniz Institute for Neurobiology, Magdeburg, Germany

13 5. Werner Siemens Imaging Center, Department of Preclinical Imaging and Radiopharmacy, Eberhard

14 Karls University Tuebingen, Germany

$16 *$ Corresponding author:

17 Bharat B. Biswal, PhD

18607 Fenster Hall, University Height

19 Newark, NJ, 07102, USA

20 bbiswal@yahoo.com

22 \# Data used in preparation of this article were obtained from the Alzheimer's Disease Neuroimaging

23 Initiative (ADNI) database (adni.loni.usc.edu). As such, the investigators within the ADNI contributed to

24 the design and implementation of ADNI and/or provided data but did not participate in analysis or writing

25 of this report. A complete listing of ADNI investigators can be found at: http://adni.loni.usc.edu/wp-

26 content/uploads/how_to_apply/ADNI_Acknowledgement_List.pdf

27

\section{Funding acknowledgements}

29 This study was funded by (US) National Institute of Health grants: R01AT009829 and R01DA038895.

31 Running headline: Causal brain metabolic connectivity in aging 


\section{Abstract}

33 During healthy brain aging, different brain regions show anatomical or functional declines at different

34 rates, and some regions may show compensatory increases in functional activity. However, few studies

35 have explored interregional influences of brain activity during the aging process. We proposed a

36 causality analysis framework combining high dimensionality independent component analysis (ICA),

37 Granger causality, and LASSO (least absolute shrinkage and selection operator) regression on

38 longitudinal brain metabolic activity data measured by Fludeoxyglucose positron emission tomography

39 (FDG-PET). We analyzed FDG-PET images from healthy old subjects, who were scanned for at least

40 five sessions with an averaged intersession interval of about year. The longitudinal data were

41 concatenated across subjects to form a time series, and the first order autoregressive model was used to

42 measure interregional causality among the independent sources of metabolic activity identified using ICA.

43 Several independent sources with reduced metabolic activity in aging, including the anterior temporal

44 lobe and orbital frontal cortex, demonstrated causal influences over many widespread brain regions. On

45 the other hand, the influenced regions were more distributed, and had smaller age related declines or even

46 relatively increased metabolic activity. The current data demonstrated interregional spreads of aging on

47 metabolic activity at the scale of a year, and have identified key brain regions in the aging process that

48 have strong influences over other regions.

49

50 Keyword: aging, anterior temporal lobe, Granger causality, LASSO regression, metabolic connectivity,

51 orbitofrontal cortex. 
52

53 The human brain undergoes development and aging across the entire life-span. Neuroimaging studies

\section{4}

55

56

57

58

59

60

61

have demonstrated that different brain regions develop and age in different rates. The global gray matter volume decreases linearly after 20s of age, but some regions such as the bilateral insula, superior parietal gyri, central sulci, and cingulate sulci show faster volumetric declines as measured by voxel-based morphometry (Good et al., 2001). Cortical thickness measures show more widespread cortical thinning patterns during aging (Salat et al., 2004). In contrast, results from functional MRI studies (fMRI) are more complex with some brain regions show increased activations in certain tasks rather than declined activations accompanied with the anatomical declines (Di, Rypma, \& Biswal, 2014; Spreng, Wojtowicz, $\&$ Grady, 2010). In addition, the functional alterations in aging may depend on the task domains and behavioral performances (Spreng et al., 2010), making it difficult to conclude a region to be functionally increased or decreased in aging. A complementary approach is to study brain activity during a state without specific behavioral involvements, i.e. resting-state. Studies have been performed earlier using positron emission tomography (PET) (Kuhl, Metter, Riege, \& Phelps, 1982; Martin, Friston, Colebatch, \& Frackowiak, 1991; Zuendorf, Kerrouche, Herholz, \& Baron, 2003), and later using resting-state fMRI (Biswal et al., 2010). Using a large sample of over 1,000 subjects, Biswal and colleagues have showed reduced resting-state activity in aging mainly in the default model network and increased activity in the visual, motor, and subcortical regions (Biswal et al., 2010).

Functionally related brain regions typically show similar co-developments (Alexander-Bloch, Raznahan, Bullmore, \& Giedd, 2013) or co-declines, therefore yielding cross-subject interregional covariances. This has been shown as early as 1980s using regional cerebral blood flow data (Prohovnik, Håkansson, \& Risberg, 1980) and regional metabolic activity data (Horwitz, Duara, \& Rapoport, 1984; Metter, Riege, Kuhl, \& Phelps, 1984) measured using PET. Later, more sophisticated methods, such as independent component analysis (ICA) and graph theory based analysis, have been applied to PET data to study the brain metabolic covariance networks (Di et al., 2017; Di \& Biswal, and Alzheimer's Disease 
Neu, 2012). Correlated metabolic activity or blood flow was typically found between left/right homotopic regions, and between some within hemisphere regions that are functionally related, e.g. language related regions in the left hemisphere. However, different connectivity patterns have been found between this metabolic covariance connectivity and the resting-state connectivity that has been typically observed from fMRI data (Di et al., 2017; Di \& Biswal, and Alzheimer's Disease Neu, 2012). The intersubject covariance patterns have also been shown using other imaging modalities, such as brain volumes (Di \& Biswal, 2016; Douaud et al., 2014; Mechelli, Friston, Frackowiak, \& Price, 2005), cortical thickness (Lerch et al., 2006), and different resting-state fMRI indices (P. A. Taylor, Gohel, Di, Walter, \& Biswal, 2012; Zhang et al., 2011).

Given the different rates of declines or relative preservations of different brain regions in aging, and large scale brain networks working in synchrony during both task execution and resting-state (Bullmore \& Sporns, 2009, 2012; Di, Gohel, Kim, \& Biswal, 2013), it is likely that the regions that are working together affect each other during the aging process. Specifically, a region that declines faster may influence another region during functional interactions in everyday basis; therefore would cause the other region to decline or show a compensatory increase of functional activity. So, it is critical to study the causal interregional influences between regions rather than the simple covariance, especially at the time scale of months to years when brain aging could be observed. Although regional brain aging is generally assumed to be linear in trend, the observed regional brain measures might showed fluctuations along the linear trend (Figure 1). The causal influence between regions could then be captured by causality analysis methods such as Granger causality (Granger, 1969). By using Granger causality we could examine whether the brain activity in a brain region at time points of months or years earlier can predict the activity of another brain region at the current time point. Granger causality at the similar time scales has been studied on brain morphological progressions in epilepsy (Zhang et al., 2017) and schizophrenia (Jiang et al., 2018) based on anatomical MRI data. However, both of these studies are cross-sectional. Large-scale multi-site longitudinal open access dataset, such as Alzheimer's Disease 
Neuroimaging Initiative (ADNI), has made it possible to examine causal influences during aging in a aging.

107

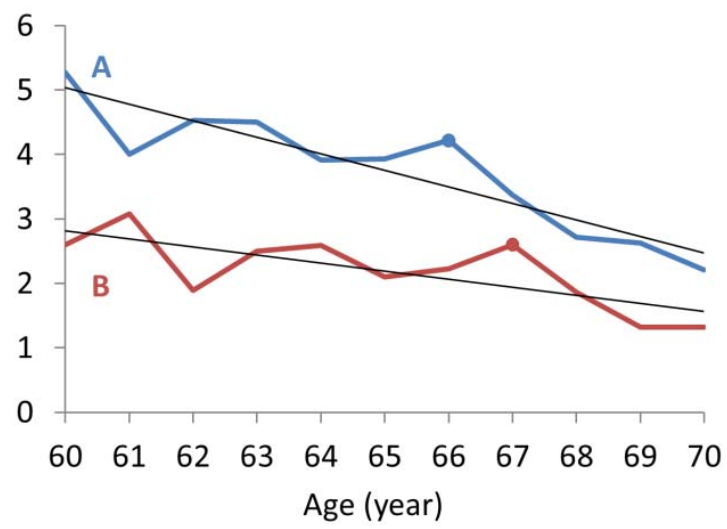

Figure 1 An illustration of interregional causal effects during aging. Regions A and B both show linear declines during aging at different rates, with additional fluctuations along the linear trends. The fluctuations of region A influences those in region $\mathrm{B}$, so that an event in $\mathrm{A}$ can be observed one time point later in B (e.g. the marked peak at age 66 and 67 in A and B, respectively).

The aim of the current study is to explore the causal interregional influences of metabolic activity during normal aging at the time scale of a year. We leveraged the longitudinal FDG-PET data from the ADNI dataset, where there were at least five sessions of FDG-PET scans for each subject at a time step of approximately one year. First, we examined regional age effects of metabolic activity to identify regions with accelerated declines, with no apparent age effects, and with relative increases. Second, we performed whole brain Granger causality analysis to identify causal influences, where the metabolic activity in a region at a certain time point can be predicted by the metabolic activity in another region at 
the previous time point. We predict that the regions that show accelerated declines during aging will

121 cause other regions to decline, thus showing interregional spreads of age effects.

123 2. Materials and methods

$124 \quad 2.1$ ADNI data

125 Data used in the preparation of this article were obtained from the ADNI database (ADNI - Alzheimer's 126 Disease Neuroimaging Initiative: RRID:SCR_003007; adni.loni.usc.edu). The ADNI was launched in

1272003 as a public-private partnership, led by Principal Investigator Michael W. Weiner, MD. The primary

128 goal of ADNI has been to test whether serial magnetic resonance imaging (MRI), positron emission

129 tomography (PET), other biological markers, and clinical and neuropsychological assessment can be combined to measure the progression of mild cognitive impairment (MCI) and early Alzheimer's disease 131 (AD). showed no signs of depression, mild cognitive impairment, or dementia, with Mini-Mental State Exam

134 (MMSE) scores between 24 and 30 and Clinical Dementia Rating (CDR) score of 0. We manually selected longitudinal FDG-PET images from the ADNI database, with participants who had at least five sessions of FDG-PET images available. As a result, 72 subjects ( 25 females) were included in the current

137 analysis with a total of 432 PET scan sessions. The numbers of available sessions ranged from 5 to 9

138 (Figure 2A). The average age at the first session was 75.8 years (62 to 86 years). For each session, we 139 calculated a mean image or adopted the only image to represent the session. The intersession interval 140 with a subject varied from 3 months to up to 8 years for a few rare cases (Figure 2B). The mean and 141 median of the intersession intervals were 1.02 and 0.98 years, respectively. 

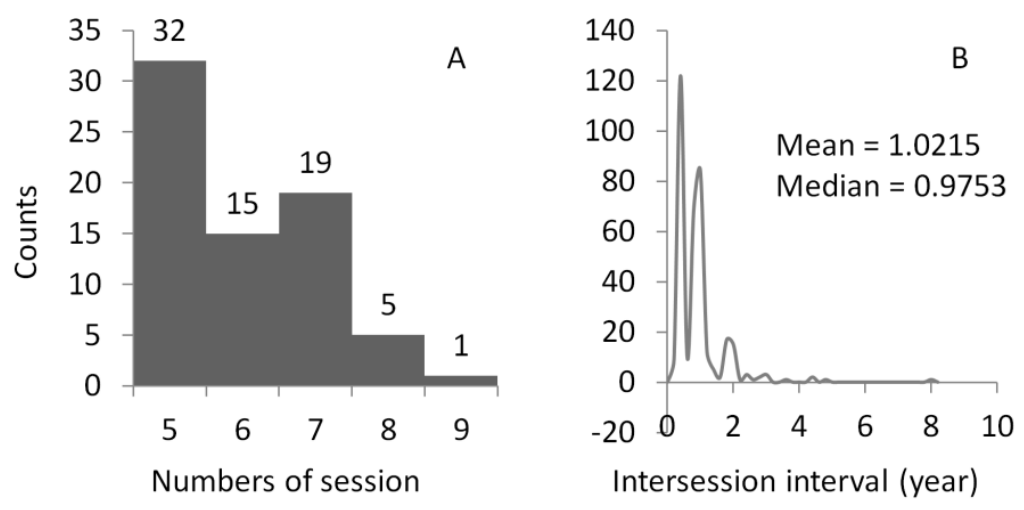

Figure 2 Histograms of the numbers of sessions for each subject (A) and the intersession intervals for all the sessions and subjects (B). The participants were typically studied at $0,6,12,24,36$ months related to the first visit, and yearly follow-ups. Therefore, the intersession intervals are likely to be around six months or one year.

The FDG-PET images were acquired from multiple sites with different PET imaging protocols.

149 However, the imaging parameters were mostly similar across different sessions within a subject. Since 150 the current analyses were all within-subject, the impacts of different imaging parameters from different 151 sites can be effectively minimized. More information about the PET protocol can be found in (Jagust et 152 al., 2010). All the images and subjects included in the current analysis can be found at: https://osf.io/4a3vt/.

\subsection{PET data preprocessing}

The PET data were preprocessed using SPM12 (SPM: RRID:SCR_007037; 
and then all the images were normalized to MNI space using the same set of parameters. We chose the resolutions of the PET images were adequate and the direct normalization has its own advantage compared with the anatomical MRI mediated method (Vince D. Calhoun et al., 2017). The images were then spatially smoothed using a Gaussian kernel with $8 \mathrm{~mm}$ FWHM (full width at half maximum). Lastly, each image was divided by its mean signal within an intracranial volume mask.

\subsection{Independent component analysis}

168 We first performed spatial ICA to separate the whole brain metabolic maps into independent sources of 169 local metabolic variations (Di \& Biswal, and Alzheimer's Disease Neu, 2012). We extracted a relatively high number of ICs, so that the resulting ICs could represent more local variations than large scale networks (Fu et al., 2018, 2019; Smith et al., 2013). This data-driven approach is an alternative to atlas-

172 based parcellation, and may be more representative to local variations of metabolic activity. The ICA was 173 performed using Group ICA of fMRI Toolbox (GIFT: RRID:SCR_001953;

174 http://mialab.mrn.org/software/gift) (V D Calhoun, Adali, Pearlson, \& Pekar, 2001). The preprocessed

175 FDG-PET images from different sessions and subjects were concatenated into a single time series, and

176 fed into the ICA analysis. Eighty one components were recommended by the minimum description

177 length (MDL) algorithm implemented in GIFT. After extraction of the 81 components, the ICs were

178 visually inspected and grouped into eight domains (Supplementary materials) as well as 21 noise

179 components. There were in total 60 ICs included in the following analysis. For each IC, the associated 180 time series were obtained to represent metabolic activity of this source in different subjects and sessions.

181 The 81 IC maps are available at: https://osf.io/4a3vt/.

\section{$182 \quad$ 2.4. Regional age effects}

183 For each subject, a general linear model (GLM) was built to examine aging effects. The GLM included

184 two regressors, a constant term and a linear age effect. The GLM analysis was performed on each IC, and 185 the $\beta$ values of the age effect were obtained. Group level analysis was then performed on each IC using a 
one sample $t$ test model to examine the group averaged effect of age. A FDR (false discovery rate)

metabolic activity, with no significant changes, and with significant decreased metabolic activity in aging.

\subsection{Interregional causality analysis}

191 We adopted Granger causality to examine the interregional causal influence of metabolic activity.

192 Specifically, we treated the longitudinal FDG-PET data as time series, and used autoregressive model to

193 predict the value of time point $\mathrm{t}$ in a region $y$ by the previous time points of another region $x$, when

194 controlling for its own previous time points. In the current data, the time step is approximately one year.

195 To account for the variability of intersession interval, the intervals between time points $t$ and $t-1$ were added as a covariate or regressed out in the analysis (see below for details). Another consideration is the order of the model, i.e. how many previous time points are used to predict the current time point. In this study, we used only the first order model to measure the causal influence of only one previous time point, which represents a time step of about one year. The limited number of time points in a subject prevents can be expressed in the following form:

$$
y_{t}=\beta_{0}+\beta_{1} \cdot y_{t-1}+\beta_{2} \cdot x_{t-1}+\varepsilon
$$

where $y$ represents the predicted time series in one brain region, and $x$ represents the predicting time series of another brain region. $y_{\mathrm{t}-1}$ represent the time series of $y_{\mathrm{t}}$ which moved one time point ahead, thus representing a autoregression model of time series $y$. The effect of interest is the predicting value of $x_{\mathrm{t}-1}$, 207 which is $\beta_{2}$.

$$
\text { We concatenated the time series across all the subjects to form a long time series for analysis }
$$

209 (Figure 3). Therefore, the model is considered fixed effect model. The time series of a subject were first $210 \mathrm{z}$ transformed to minimize inter-subject variation, where $\mathrm{m}_{\mathrm{i}}$ represents the total number of time points in a 
subject i. For each subject, we included the time points 2 to $\mathrm{m}$ of the time series of a region as the

212 predicted variable $y_{\mathrm{t}}$. The autoregressive variable $y_{\mathrm{t}-1}$ included the time points 1 to $\mathrm{m}-1$ of the time

213 series of the same region. The predicting variable $x_{\mathrm{t}-1}$ was the time points 1 to $\mathrm{m}-1$ from another region

$214 x$. After concatenation, there were in total 360 data points in the time series.

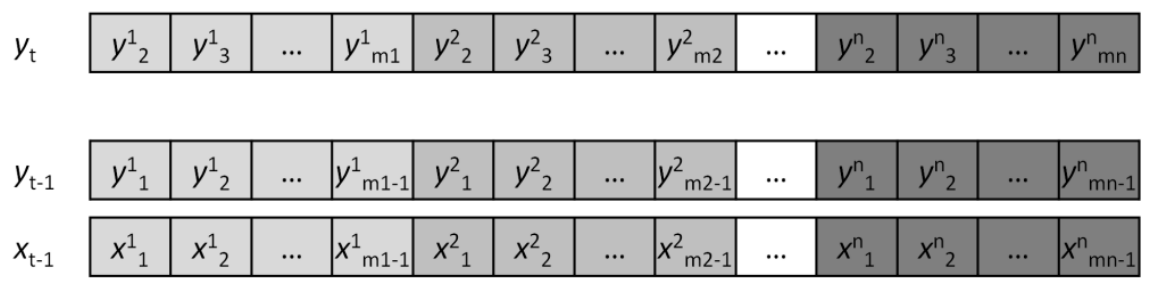

215

Figure 3 Illustration of the construction of the variables used in the causality analysis. X represents the predicting region, and y represents the predicted region. The superscript represents different subjects, with a total number of $\mathrm{n}$. The subscript represents the scan session in a subject, with a total number of mi for a subject $\mathrm{i}$. between time $t$ and time $t-1$ were included in the model as a covariate. We first performed such analysis on each pair of ICs to obtain the predicting effect $\left(\beta_{2}\right)$ and corresponding $\mathrm{p}$ values, which formed a $60 \mathrm{x}$

22460 matrix of causal effects. FDR correction at $\mathrm{p}<0.05$ was used to correct for multiple comparisons of 225 the in total 3,540 (60 x 59) effects, where autoregressive effects along the diagonal were not tested. then as following for a predicted region $x^{\mathrm{i}}$ :

$$
x_{t}^{i}=\beta_{0}+\beta_{1} \cdot x_{t-1}^{1}+\beta_{2} \cdot x_{t-1}^{2}+\beta_{3} \cdot x_{t-1}^{3}+\ldots+\beta_{p} \cdot x_{t-1}^{p}+\varepsilon
$$


231 Since all the ICs were included in the model, there was no need to differentiate the variables of $x$ and $y$.

232 Therefore, we use $x$ to denote all the time series variables. The superscripts of $x$ now represent different

233 ICs, where p represents the total number of the IC. Before entering in to the model, a time series

234 representing the intersession interval between time point t and $\mathrm{t}-1$ were regressed out from all the $x_{\mathrm{t}-1}$

235 time series to account for the intersession interval variability. Estimating the multivariate model may be

236 challenging, especially when some of the IC time series may be highly correlated. It can be assumed that

237 only a small number of ICs may influence the predicted IC. In this scenario, one can use regularization

238 method to estimate the sparse influence effects, such as using LASSO (least absolute shrinkage and

239 selection operator) (Tang, Bressler, Sylvester, Shulman, \& Corbetta, 2012; Tibshirani, 1996). The

240 motivation of choosing LASSO over other regularization methods is that the LASSO regularization can

241 force some parameters in the model to be zero thus resulting in only a small number of non-zero

242 parameters. This is important in the current context, because the aim is to identify a small number of

243 interregional influences. Since this model examines the prediction of the time series of one IC by the

244 time series of all the other ICs, the analysis only needed to be performed for 60 times (compared with 60

$245 \times 59$ times in the pair-wise analysis) to cover all the ICs.

The LASSO regression was performed using the lasso function implemented in MATLAB. To

247 determine an optimal regularization factor $\lambda$, we used a set of $\lambda$ from 0 (no regularization) to 0.5 with a

248 step of 0.001 . The identified non-zero influences dropped dramatically as the increase of $\lambda$. We identify

249 the $\lambda$ where the number of non-zero influences were the closest to the number of significant effects when

250 using FDR correction in the pair-wise analysis, and reported all the non-zero influencing effects.

The resulting $60 \times 60$ influencing matrix can be treated as a directed network graph, where the

252 ICs represent the nodes and the causal influences represent directed edges of the graph. We calculated in-

253 degree and out-degree of the 60 nodes to characterize the importance of an IC in the whole brain

254 influencing graph. To ensure that the degree calculation was not affected by arbitrary defined threshold,

255 we also explored the graphs from other $\lambda$ values to verify the identified hub regions are still present. To 
visualize the network topology, we identified the giant component where all the nodes in the component were somehow connected (without considering the direction of the influences). The giant components were visualized using the force layout.

\section{Results}

\subsection{Age effect on regional metabolic activity}

262 We first examined the age effects on the regional metabolic activity for the 60 ICs. Statistical significant

263 ICs at $p<0.05$ after FDR correction are shown in Figure 4. Eighteen ICs showed significant reduced

264 metabolic activity, including one IC that covered the inferior portion of the cerebellum (IC 1), three ICs

265 that coved visual cortex (IC 17, 32, and 53), three ICs that covered the posterior parietal cortex (IC 21, 27,

266 and 28), five ICs that covered the anterior portion of the temporal lobe and insula (IC 24, 44, 63, 66, and

267 77), one IC that covered the thalamus and basal ganglia (IC 23), two ICs that covered the orbital frontal

268 cortex and frontal pole (IC 3 and 48), and three ICs that covered the cingulate cortex and neighboring

269 midline cortical regions (IC 7, 37, and 46). The left panel of Figure 5 illustrates the negative age effects

270 of an example IC (IC 48). It can be seen that there is a general linear trend of decrease of metabolic

271 activity. But each subject showed fluctuations of metabolic activity along the linear trend. In contrast, 6

272 ICs showed increased metabolic activity. It should be noted that due to the nature of PET imaging, the

273 global signal for each PET image has to be normalized. Therefore, it is difficult to say whether the

274 positive age effect represents increased metabolic activity, or a relative increase with reference to the

275 global effect. The ICs with relative increased metabolic activity during aging included one IC covering

276 the inferior and posterior portion of the cerebellum (IC 13), two subcortical ICs covering the basal ganglia,

277 insula, amygdala, and thalamus (IC 19 and 52), and three ICs of sensorimotor regions (IC 36, 50, and 68).

278 The right panel of Figure 5 illustrates the positive age effects of an example IC (IC 19). There were 36

279 ICs that did not show statistically significant age effects at $\mathrm{p}<0.05$ after FDR correction. The middle 
281 effect.
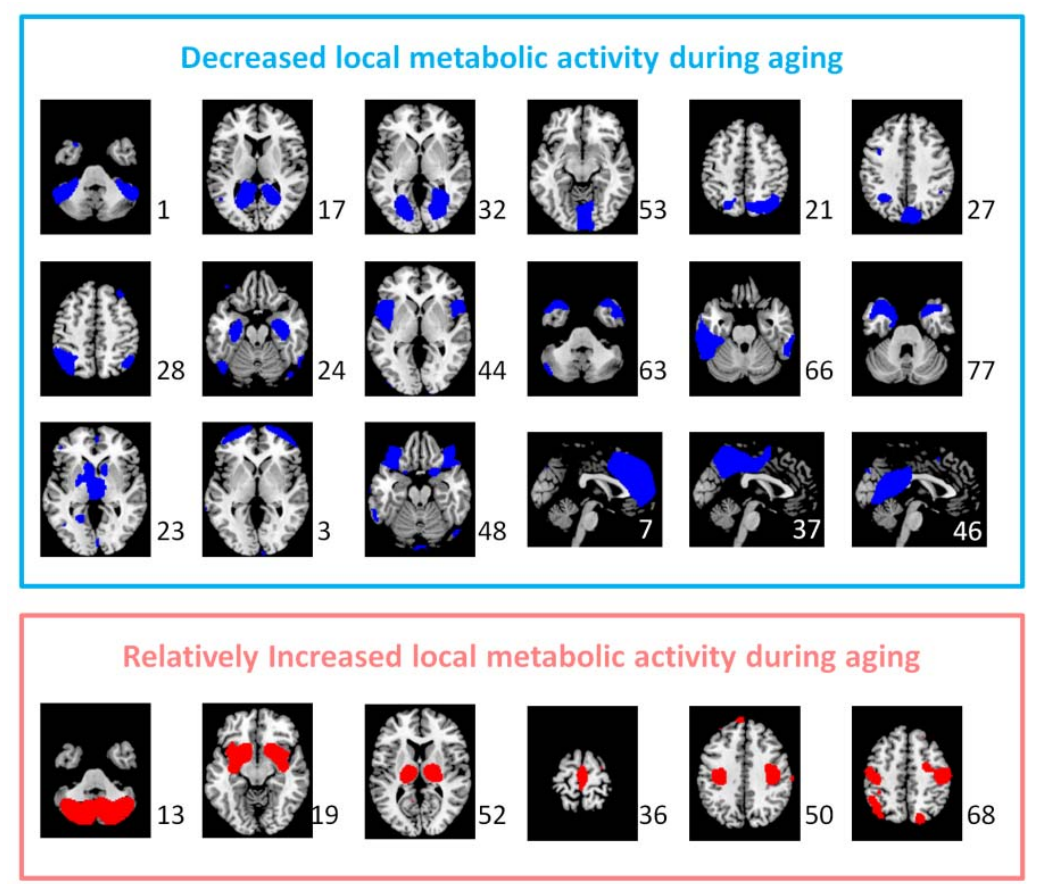

Figure 4 The independent components (ICs) that showed statistically significant decreased (blue) and increased (red) metabolic activity during aging after controlling for global effect at $\mathrm{p}<0.05$ with false discovery rate (FDR) correction. The numbers to the bottom right represent the IC number.

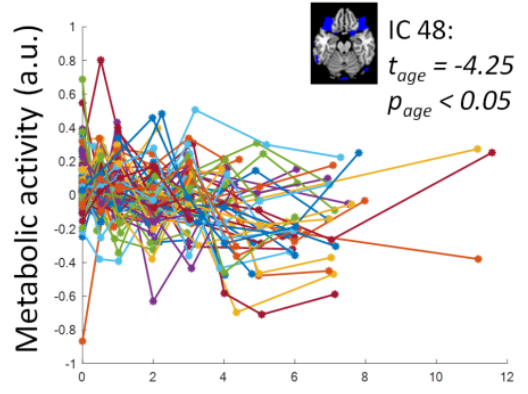

\section{8}
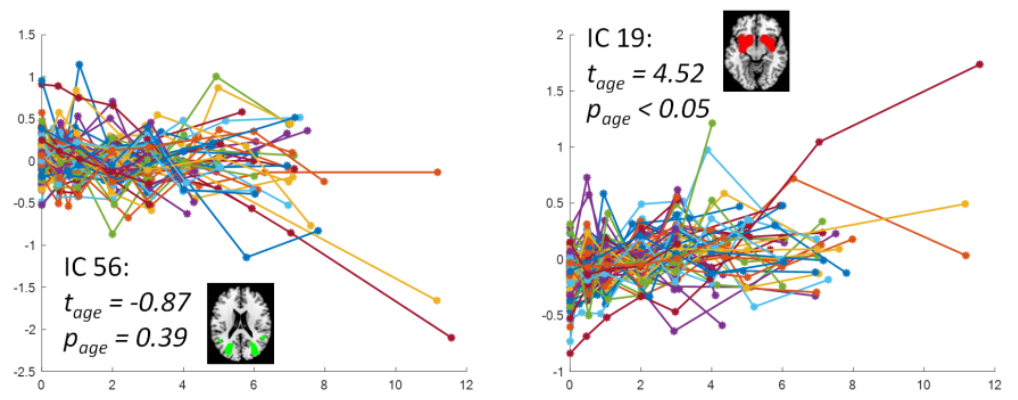

Time since first scan (year)

Figure 5 Examples of aging effects of metabolic activity of three independent components (ICs) that had negative, non-significant, and positive aging effects. Each colored line represents one subject. T and $\mathrm{p}$ values represent group-level one sample t test statistics. A.u., arbitrary unit. 


\subsection{Interregional causal influences of metabolic activity}

293 We first applied pairwise autoregressive model to obtain a 60 x 60 matrix of the interregional causal 294 influences of metabolic activity between each pair of the ICs (Figure 6A). When using a statistical 295 threshold of $\mathrm{p}<0.05$ of FDR correction, 14 positive and 13 negative causal influences were identified

296 (Figure 6B). We next performed LASSO regression with $x_{\mathrm{t}}$ of an IC as the predicted variable and $x_{\mathrm{t}-1}$ of 297 all the ICs as the predicting variables using a range of $\lambda$. We identified the $\lambda$ value where the number of 298 non-zero effects was the closest to the number of significant effects in the pairwise analysis. The 299 resulting influencing effects at $\lambda=0.162$ (Figure 6C) look in general similar to the significant effects 300 identified by the pairwise analysis, although some subtle differences can be noted. There were 15 positive and 13 negative causal influences identified using LASSO regression (Table 1).

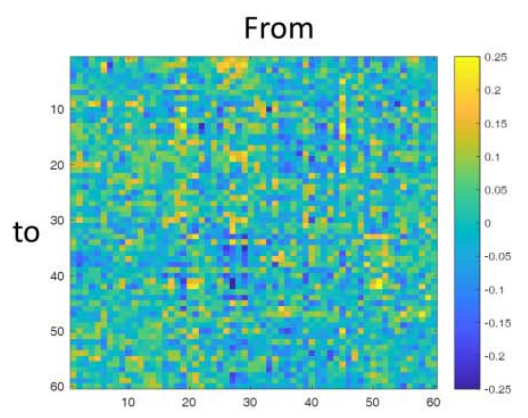

A. Pairwise: Raw

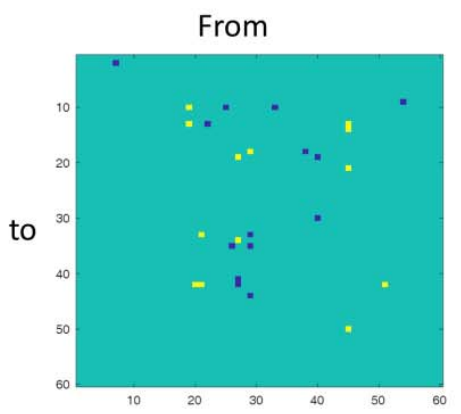

B. Pairwise: $p<0.05$ FDR

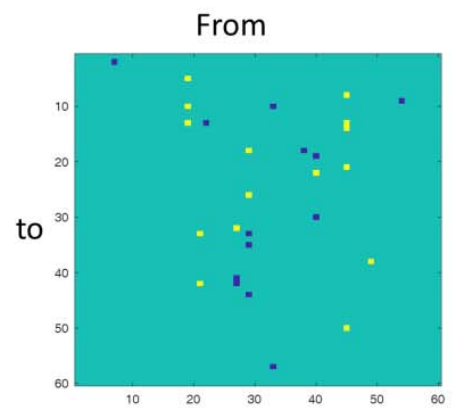

C. LASSO: $\lambda=0.162$

Figure 6 A, Pairwise matrix of interregional causal influence of metabolic activity. The columns

represent influencing independent components (ICs), while the rows represent influenced ICs. B, Ternary matrix of significant positive or negative interregional causal influences thresholded at $\mathrm{p}<0.05$ after false discovery rate (FDR) correction. C, Ternary matrix of positive or negative interregional causal influences identified at $\lambda=0.162$ using LASSO (least absolute shrinkage and selection operator) regression.

Among the 28 causal influences from LASSO regression, the first giant component was comprised of 26 causal influences involving 25 ICs (Figure 7). The IC maps were color coded based on 
bioRxiv preprint doi: https://doi.org/10.1101/490292; this version posted June 25, 2019. The copyright holder for this preprint (which was not certified by peer review) is the author/funder, who has granted bioRxiv a license to display the preprint in perpetuity. It is made available under aCC-BY-NC-ND 4.0 International license.

311 their regional age effects to illustrate the relationships between regional metabolic activity changes and

312 the signs of causal influences. It can be seen that the influences between two decreased regions or two

313 increased regions in aging were in general positive, but the influences between one increased and one

314 decreased regions were in general negative. For example, the bilateral anterior temporal IC (IC\# 63 in

315 Figure 6) positively influenced the medial parietal IC (IC\# 27), but negatively influenced the basal

316 ganglia IC (IC\# 19). It is consistent with the direction of the spread of age effects. There were also ICs

317 that without apparent age effects, where the signs of causal influences with other regions did not show

318 clear pattern.

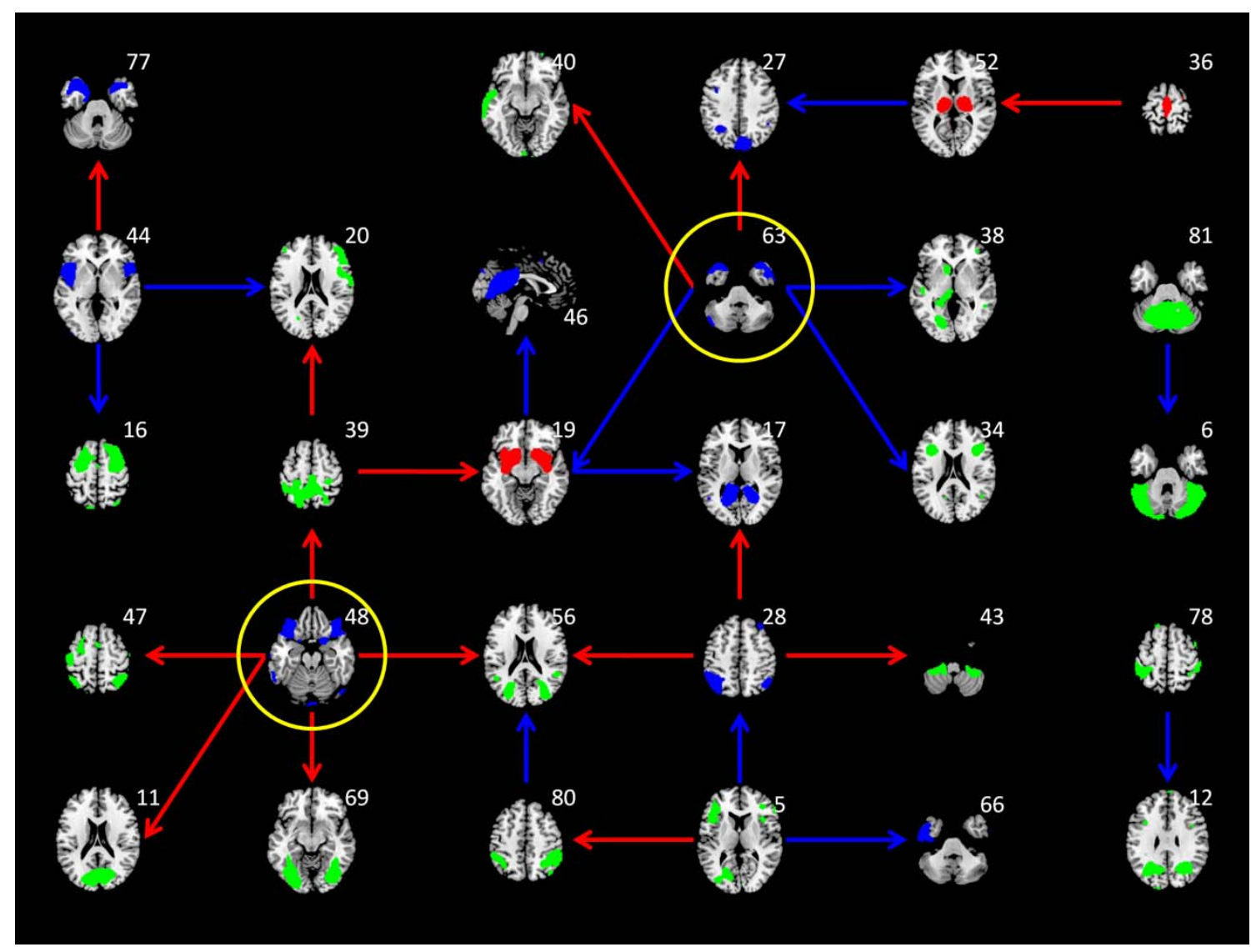

320 Figure 7 Interregional causal influences network at $\lambda$ of 0.162 using LASSO (least absolute shrinkage and

321 selection operator) regression. The colors of the independent component maps represent increased (red),

322 decreased (blue), and non-significant (green) age effects on metabolic activity. The colors of the arrows 
represent positive (red) and negative (blue) interregional influences, respectively. The maps highlighted with yellow circle represent hub regions in the network.

327 regions that are more influencing or influenced to other regions, we plotted the first giant components of 328 the interregional influencing network using force layout at $\lambda=0.162$, and also at more liberal thresholds 329 of $\lambda=0.142$ and $\lambda=0.122$ (Figure 8). The node sizes represent the out-degree or in-degree of a node in

330 the network in the upper and middle panels, respectively. It can be seen that the nodes with large out-

331 degree were in general the regions with decreased metabolic activity (blue nodes). The red arrows

332 highlighted the two nodes that had 5 out-degrees at $\lambda=0.162$ and remained among the highest out-degree 333 nodes at the lower $\lambda$ values. These two nodes were also highlighted in Figure 7 , which covered the 334 bilateral orbitofrontal cortex (IC\# 48) and the bilateral anterior temporal lobe (IC\# 63). While in terms of 335 in-degree, there were no clear regions that had exceptionally high in-degree compared with other nodes.

336 The node with high in-degree had no apparent age effects (green nodes) or had increased metabolic

337 activity with age (brown nodes). The distributions of nodal out- and in-degree confirmed that the out-

338 degree distributions had heavy tailed distributions compared with the in-degree distributions (lower 339 panels in Figure 8). 

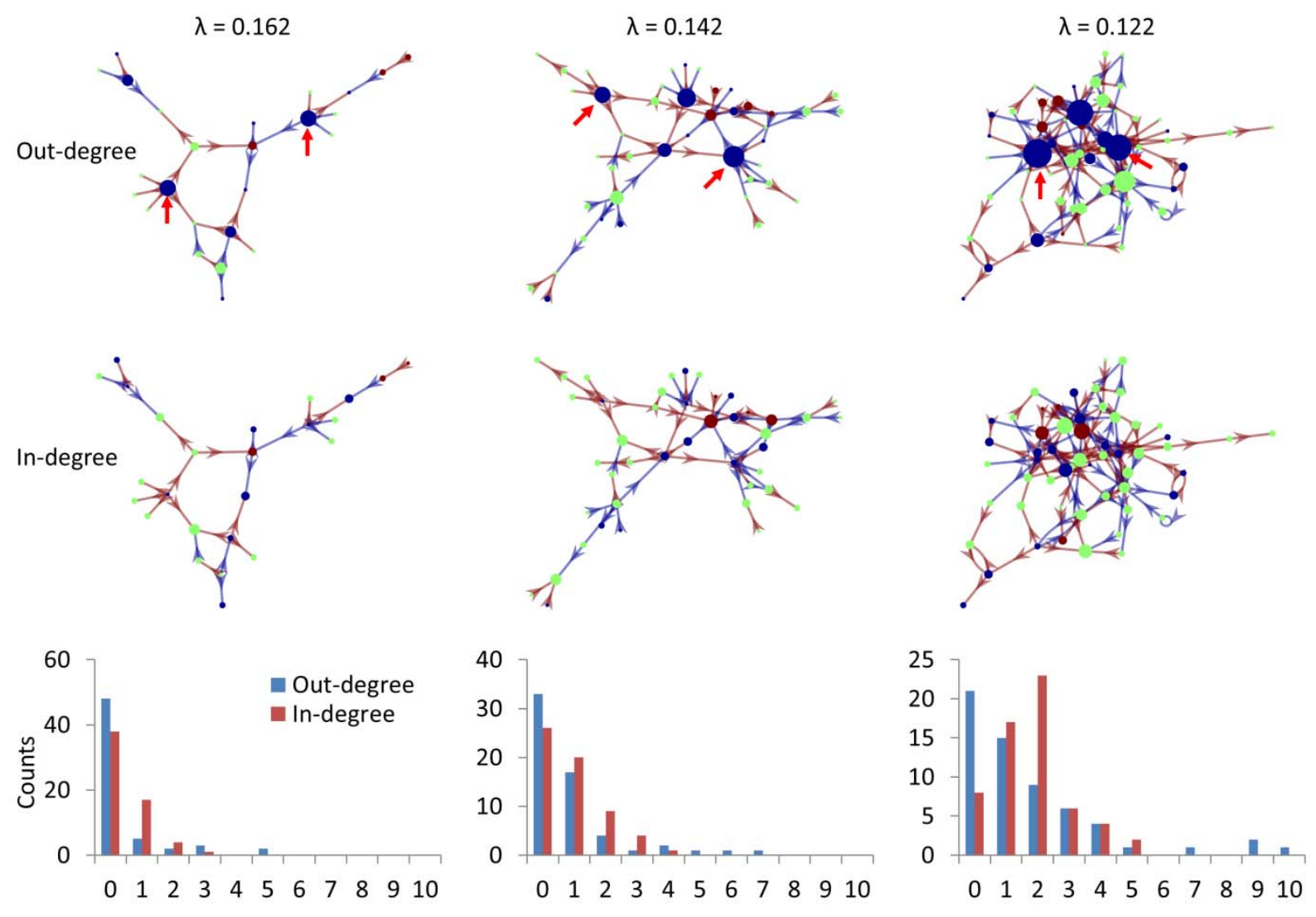

Degree

Figure 8 The giant component of the causal interregional influencing network of metabolic activity at different $\lambda$ levels with the sizes of the nodes reflecting out-degree (top row) and in-degree (middle row).

The bottom row shows the degree distributions of the whole influencing network at the three $\lambda$ levels.

Brown and blue arrows indicate positive and negative influences identified using LASSO regression.

Blue, green, and brown regions indicate positive, none, and negative age effects on regional metabolic activity. The red arrows highlight the two influencing nods at different $\lambda$ levels.

\section{Discussion}

By applying autoregressive model on longitudinal FDG-PET data, the current study demonstrated causal 
influenced many other ICs. In contrast, the influenced ICs were more widespread and with less local aging effects or even with relatively increased metabolic activity. To the best of our knowledge, this is the first study to demonstrate longitudinal interregional causal influences of brain activity during aging at the time scale of a year. had decreased metabolic activity. On the other hand, the influenced regions were not restricted to the 359 regions with reduced metabolic activity in aging. Indeed, the ICs that had relatively greater in-degree 360 values than other ICs were usually without apparent age effects, or even with relatively increased 361 metabolic activity, e.g. the basal ganglia and thalamus. Therefore, the causal interregional influences in general reflected the spread of age effects from brain regions that had already declined to regions that are declining or relatively preserved. We note that the absence of regional age effects should be interpreted with caution, because the removal of global effects during calculation of regional age effects could have removed significant age effects that were similar to the global effects.

367 and the regional age effects. A positive influence indicates that the metabolic activity in region A at the current time point positively predicts the metabolic activity in region B at the next time point. While a negative influence indicates a negative prediction. If the two regions are both decreasing during aging, then a positive influence may indicate a spread of metabolic activity decline between the two regions. On

371 the other hand, if region A decreases but region B shows relatively increased metabolic activity, and there

372 is a negative influence between $\mathrm{A}$ and $\mathrm{B}$, then it may indicate a compensation of region $\mathrm{B}$ that is resulted

373 from the declined function of region A. A close look at the patterns of the directions of the local and

374 interregional effects indicated that most of the effects observed were consistent with the spatial spread or

375 compensation interpretations. That is, the influences between two decreased regions were all positive,

376 and the influences between one decreased region and one increased regions were all negative. 
The current analysis identified several hubs that influenced other brain regions, most prominently

the anterior temporal lobe and orbital frontal cortex. The anterior temporal lobe (IC 63) is connected to several major white matter tracts such the cingulum, inferior longitudinal fasciculus, and uncinate fasciculus (Catani \& Thiebaut de Schotten, 2008), which could support its influencing role to other regions such as the subcortical regions, inferior frontal cortex, and left temporal cortex. To better characterize its functional correlates, we submitted the IC map into NeuroVault (NeuroVault, RRID:SCR_003806; https://neurovault.org), and decoded the functions of these maps using large-scale meta-analytic data from Neurosynth (NeuroSynth, RRID:SCR_006798; http://neurosynth.org/) (Rubin et al., 2017). The first five functional terms were all about language and semantic processing (See Supplementary Table S2). Studies also showed that electrical stimulation of the anterior temporal lobe can improve proper name recalls in aging (Ross, McCoy, Coslett, Olson, \& Wolk, 2011), and bilingualism can protect the integrity of anterior temporal lobe in aging (Abutalebi et al., 2014). Taken together, the results suggest that language process might be an important factor modulating brain aging. The orbital frontal cortex (IC 48) is connected to the uncinate fasciculus and inferior frontooccipital fasciculus (Catani \& Thiebaut de Schotten, 2008), which could support its influences to the posterior visual regions. The functional words related to the orbitofrontal IC were mainly about emotional processing (Supplementary Table S2). In older population, smaller orbitofrontal volumes are shown to be associated with depression (Lai, Payne, Byrum, Steffens, \& Krishnan, 2000; W. D. Taylor et al., 2003). Taken together, emotional process might also be an important factor modulating brain aging. However, although previous studies have shown associations between resting-state brain activity and task activations (Di, Kannurpatti, Rypma, \& Biswal, 2013; Yuan et al., 2013), the extent to what resting-state brain activity can reflect certain brain functions are still largely unknown. Further studies might need to design proper tasks to better link functions to brain activations.

A limitation of the current analysis is the potential confounding effect due to partial volume (Bonte et al., 2017; Rousset, Ma, \& Evans, 1998), i.e. whether an observed effect is due to the changes of 
bona fide metabolic activity or the changes of underlying gray matter volume. However, the following

403

404

405

406

407

408

409

410

411

412

413

414

415

416

417

418

419

420

421

422

423

424

425

426

reasons make the partial volume confounding less problematic. First, the current analysis adopted within subject comparison, which has already minimized the partial volume effects due to inter subject anatomical variability. Second, we applied ICA analysis to identify independent sources of metabolic variability. Some components that were likely due to enlargement of ventricle and are spatially overlapped with the included ICs, have been already removed. For example, there was an IC largely located in ventricle area (IC 79 in supplementary Figure S9) but with substantial overlaps with the ICs of the thalamus and basal ganglia (supplementary Figure S5). The IC 79 had the second strongest negative age effect among all the ICs. The included ICs that had spatial overlap with this IC showed no age effects or even positive age effects, suggesting that the partial volume effects associated with enlarged ventricle have been minimized in these ICs. Third, even though the observed causal influences may still somehow contributed by the residual partial volume effects, the causal influences of volumetric reductions may still be important findings for understanding brain aging. The structural MRI images are available in the ADNI dataset, but were not always acquired at the same time point as the PET images, making the incorporation of MRI images in the model difficult. Future studies should certainly consider taking into account of anatomical information in the analysis. Indeed, it may be theoretically more important to study the interaction or causal influences between brain anatomy and functions in aging. According to the compensation model, the reduction of gray matter will lead to elevated functional responses, which then give rise to less affected behavioral performances (Gregory et al., 2018; Reuter-Lorenz \& Park, 2014; Shafto \& Tyler, 2014). A direct examination of causal influences among local and interregional gray matter structures, functions, and behavioral performances may provide more insight to the dynamic of compensation process in aging.

One strength of the current analysis approach is that we adopted multivariate methods and LASSO to include all the ICs in the predicting models, which in theory can prevent identifying ICs that have indirect predicting effects to the target (Smith et al., 2011; Tang et al., 2012). On the other hand, 
there are also several simplifications of the Granger causality analysis, such as the inclusion of only the

first order model and the assumption of equal time steps. Since the current study is the first to explore the

430 further studies. But practically due to the limited availability of longitudinal data, this question is difficult

431 to solve at the current stage. Regarding the variable time steps of the time series, we added the

432 intersession interval as a covariate to minimize the effects, which is similar to a previous work (Jiang et

433 al., 2018). More sophisticated models, such as generative model and differential equation based method

434 (G. Ziegler, Penny, Ridgway, Ourselin, \& Friston, 2015; Gabriel Ziegler, Ridgway, Blakemore,

435 Ashburner, \& Penny, 2017), may be used in future to better characterize the causal effects.

\section{Conclusion}

By applying Granger causality analysis on longitudinal FDG-PET images of healthy old participants at a time step of one year, the current analysis revealed interregional causal influences during aging. Several regions with reductions in local metabolic activity during aging, including the bilateral anterior temporal

441 lobe and orbitofrontal cortex, showed causal influences to other regions, supporting an interregional

442 spread of age effects in the brain. The current analysis and results could add new insights to the

443 neurocognitive aging literature about interregional interactions during the aging process.

\section{Acknowledgements}

446 Data collection and sharing for this project was funded by the Alzheimer's Disease Neuroimaging

447 Initiative (ADNI) (National Institutes of Health Grant U01 AG024904) and DOD ADNI (Department of

448 Defense award number W81XWH-12-2-0012). ADNI is funded by the National Institute on Aging, the

449 National Institute of Biomedical Imaging and Bioengineering, and through generous contributions from

450 the following: AbbVie, Alzheimer's Association; Alzheimer's Drug Discovery Foundation; Araclon

451 Biotech; BioClinica, Inc.; Biogen; Bristol-Myers Squibb Company; CereSpir, Inc.; Cogstate; Eisai Inc.; 
Elan Pharmaceuticals, Inc.; Eli Lilly and Company; EuroImmun; F. Hoffmann-La Roche Ltd and its affiliated company Genentech, Inc.; Fujirebio; GE Healthcare; IXICO Ltd.; Janssen Alzheimer

454 Immunotherapy Research \& Development, LLC.; Johnson \& Johnson Pharmaceutical Research \&

455 Development LLC.; Lumosity; Lundbeck; Merck \& Co., Inc.; Meso Scale Diagnostics, LLC.; NeuroRx

456 Research; Neurotrack Technologies; Novartis Pharmaceuticals Corporation; Pfizer Inc.; Piramal Imaging;

457 Servier; Takeda Pharmaceutical Company; and Transition Therapeutics. The Canadian Institutes of

458 Health Research is providing funds to support ADNI clinical sites in Canada. Private sector contributions

459 are facilitated by the Foundation for the National Institutes of Health (www.fnih.org). The grantee

460 organization is the Northern California Institute for Research and Education, and the study is coordinated

461 by the Alzheimer's Therapeutic Research Institute at the University of Southern California. ADNI data

462 are disseminated by the Laboratory for Neuro Imaging at the University of Southern California.

464 Author contributions:

465 X.D. conceived the idea, developed and performed the data analysis. All authors discussed the results, and contributed to the final manuscript.

\section{Conflict of interest statement:}

469 The authors declare that there is no conflict of interest regarding the publication of this article.

\section{Supplemental materials:}

472 Supplemental materials include one word file containing nine supplementary Figures and two

473 supplementary Tables. Other supporting information can be found at https://osf.io/4a3vt/.

\section{Reference:}

476 Abutalebi, J., Canini, M., Della Rosa, P. A., Sheung, L. P., Green, D. W., \& Weekes, B. S. (2014). 
Bilingualism protects anterior temporal lobe integrity in aging. Neurobiology of Aging, 35(9), 21262133. http://doi.org/10.1016/j.neurobiolaging.2014.03.010

Alexander-Bloch, A., Raznahan, A., Bullmore, E., \& Giedd, J. (2013). The convergence of maturational change and structural covariance in human cortical networks. The Journal of Neuroscience $\square$ : The Official Journal of the Society for Neuroscience, 33(7), 2889-99. http://doi.org/10.1523/JNEUROSCI.3554-12.2013

Biswal, B. B., Mennes, M., Zuo, X.-N., Gohel, S., Kelly, C., Smith, S. M., ... Milham, M. P. (2010). Toward discovery science of human brain function. Proceedings of the National Academy of Sciences of the United States of America, 107(10), 4734-9. http://doi.org/10.1073/pnas.0911855107

Bonte, S., Vandemaele, P., Verleden, S., Audenaert, K., Deblaere, K., Goethals, I., \& Van Holen, R. (2017). Healthy brain ageing assessed with 18F-FDG PET and age-dependent recovery factors after partial volume effect correction. European Journal of Nuclear Medicine and Molecular Imaging, 44(5), 838-849. http://doi.org/10.1007/s00259-016-3569-0

Bullmore, E., \& Sporns, O. (2009). Complex brain networks: graph theoretical analysis of structural and functional systems. Nature Reviews. Neuroscience, 10(3), 186-98. http://doi.org/10.1038/nrn2575

Bullmore, E., \& Sporns, O. (2012). The economy of brain network organization. Nature Reviews Neuroscience, 13(5), 336-349. http://doi.org/10.1038/nrn3214

Calhoun, V. D., Adali, T., Pearlson, G. D., \& Pekar, J. J. (2001). A method for making group inferences from functional MRI data using independent component analysis. Human Brain Mapping, 14(3), 140-51. Retrieved from http://www.ncbi.nlm.nih.gov/pubmed/11559959

Calhoun, V. D., Wager, T. D., Krishnan, A., Rosch, K. S., Seymour, K. E., Nebel, M. B., ... Kiehl, K. (2017). The impact of T1 versus EPI spatial normalization templates for fMRI data analyses. Human Brain Mapping, 38(11), 5331-5342. http://doi.org/10.1002/hbm.23737

Catani, M., \& Thiebaut de Schotten, M. (2008). A diffusion tensor imaging tractography atlas for virtual in vivo dissections. Cortex; a Journal Devoted to the Study of the Nervous System and Behavior, 
Di, X., \& Biswal, and Alzheimer's Disease Neu, B. B. (2012). Metabolic Brain Covariant Networks as Revealed by FDG-PET with Reference to Resting-State fMRI Networks. Brain Connectivity, 2(5), 275-283. http://doi.org/10.1089/brain.2012.0086

Di, X., \& Biswal, B. B. (2016). Similarly Expanded Bilateral Temporal Lobe Volumes in Female and Male Children With Autism Spectrum Disorder. Biological Psychiatry: Cognitive Neuroscience and Neuroimaging, 1(2), 178-185. http://doi.org/10.1016/j.bpsc.2015.11.006

Di, X., Gohel, S., Kim, E. H., \& Biswal, B. B. (2013). Task vs. rest-different network configurations

Di, X., Gohel, S., Thielcke, A., Wehrl, H. F., Biswal, B. B., \& Alzheimer's Disease Neuroimaging Initiative. (2017). Do all roads lead to Rome? A comparison of brain networks derived from intersubject volumetric and metabolic covariance and moment-to-moment hemodynamic correlations in old individuals. Brain Structure \& Function, 222(8), 3833-3845. http://doi.org/10.1007/s00429$017-1438-7$

Di, X., Kannurpatti, S. S., Rypma, B., \& Biswal, B. B. (2013). Calibrating BOLD fMRI Activations with Neurovascular and Anatomical Constraints. Cerebral Cortex (New York, N.Y. $\square:$ 1991), 23(2), 25563. http://doi.org/10.1093/cercor/bhs001

Di, X., Rypma, B., \& Biswal, B. B. (2014). Correspondence of executive function related functional and anatomical alterations in aging brain. Progress in Neuro-Psychopharmacology \& Biological (2014). A common brain network links development, aging, and vulnerability to disease. Proceedings of the National Academy of Sciences, 111(49), 17648-17653. http://doi.org/10.1073/pnas.1410378111 
527

528

529

530

531

532

533

534

535

536

537

538

539

540

541

542

543

544

545

546

547

548

549

550

551

Fu, Z., Tu, Y., Di, X., Du, Y., Pearlson, G. D., Turner, J. A., ... Calhoun, V. D. (2018). Characterizing dynamic amplitude of low-frequency fluctuation and its relationship with dynamic functional connectivity: An application to schizophrenia. NeuroImage, 180, 619-631. http://doi.org/10.1016/j.neuroimage.2017.09.035

Fu, Z., Tu, Y., Di, X., Du, Y., Sui, J., Biswal, B. B., ... Calhoun, V. D. (2019). Transient increased thalamic-sensory connectivity and decreased whole-brain dynamism in autism. NeuroImage, 190, 191-204. http://doi.org/10.1016/j.neuroimage.2018.06.003

Good, C. D., Johnsrude, I. S., Ashburner, J., Henson, R. N., Friston, K. J., \& Frackowiak, R. S. (2001). A voxel-based morphometric study of ageing in 465 normal adult human brains. NeuroImage, 14(1 Pt 1), 21-36. http://doi.org/10.1006/nimg.2001.0786

Granger, C. W. J. (1969). Investigating Causal Relations by Econometric Models and Cross-spectral Methods. Econometrica, 37(3), 424. http://doi.org/10.2307/1912791

Gregory, S., Long, J. D., Klöppel, S., Razi, A., Scheller, E., Minkova, L., ... Orth, M. (2018). Testing a longitudinal compensation model in premanifest Huntington's disease. Brain. http://doi.org/10.1093/brain/awy122

Horwitz, B., Duara, R., \& Rapoport, S. I. (1984). Intercorrelations of Glucose Metabolic Rates Between Brain Regions: Application to Healthy Males in a State of Reduced Sensory Input. Journal of Cerebral Blood Flow \& Metabolism, 4(4), 484-499. http://doi.org/10.1038/jcbfm.1984.73

Jagust, W. J., Bandy, D., Chen, K., Foster, N. L., Landau, S. M., Mathis, C. A., .. Koeppe, R. A. (2010). The Alzheimer's Disease Neuroimaging Initiative positron emission tomography core. Alzheimer's \& Dementia, 6(3), 221-229. http://doi.org/10.1016/j.jalz.2010.03.003

Jiang, Y., Luo, C., Li, X., Duan, M., He, H., Chen, X., .. Yao, D. (2018). Progressive Reduction in Gray Matter in Patients with Schizophrenia Assessed with MR Imaging by Using Causal Network Analysis. Radiology, 287(2), 633-642. http://doi.org/10.1148/radiol.2017171832

Kuhl, D. E., Metter, E. J., Riege, W. H., \& Phelps, M. E. (1982). Effects of Human Aging on Patterns of 
Local Cerebral Glucose Utilization Determined by the [ 18 F] Fluorodeoxyglucose Method. Journal of Cerebral Blood Flow \& Metabolism, 2(2), 163-171. http://doi.org/10.1038/jcbfm.1982.15

Lai, T.-J., Payne, M. E., Byrum, C. E., Steffens, D. C., \& Krishnan, K. R. R. (2000). Reduction of orbital frontal cortex volume in geriatric depression. Biological Psychiatry, 48(10), 971-975. http://doi.org/10.1016/S0006-3223(00)01042-8

Lerch, J. P., Worsley, K., Shaw, W. P., Greenstein, D. K., Lenroot, R. K., Giedd, J., \& Evans, A. C. (2006). Mapping anatomical correlations across cerebral cortex (MACACC) using cortical thickness

\section{0}

561

562

563

564

565

566

567

568

569

570

571

572

573

574

575

576 from MRI. NeuroImage, 31(3), 993-1003. http://doi.org/10.1016/j.neuroimage.2006.01.042

Martin, A. J., Friston, K. J., Colebatch, J. G., \& Frackowiak, R. S. J. (1991). Decreases in Regional Cerebral Blood Flow with Normal Aging. Journal of Cerebral Blood Flow \& Metabolism, 11(4), 684-689. http://doi.org/10.1038/jcbfm.1991.121

Mechelli, A., Friston, K. J., Frackowiak, R. S., \& Price, C. J. (2005). Structural covariance in the human cortex. The Journal of Neuroscience $\square$ : The Official Journal of the Society for Neuroscience, 25(36), 8303-10. http://doi.org/10.1523/JNEUROSCI.0357-05.2005

Metter, E. J., Riege, W. H., Kuhl, D. E., \& Phelps, M. E. (1984). Cerebral Metabolic Relationships for Selected Brain Regions in Healthy Adults. Journal of Cerebral Blood Flow \& Metabolism, 4(1), 1-7. http://doi.org/10.1038/jcbfm.1984.1

Prohovnik, I., Håkansson, K., \& Risberg, J. (1980). Observations on the functional significance of regional cerebral blood flow in "resting" normal subjects. Neuropsychologia, 18(2), 203-17. http://doi.org/10.1016/0028-3932(80)90066-4

Reuter-Lorenz, P. A., \& Park, D. C. (2014). How Does it STAC Up? Revisiting the Scaffolding Theory of Aging and Cognition. Neuropsychology Review, 24(3), 355-370. http://doi.org/10.1007/s11065-0149270-9

Ross, L. A., McCoy, D., Coslett, H. B., Olson, I. R., \& Wolk, D. A. (2011). Improved Proper Name Recall in Aging after Electrical Stimulation of the Anterior Temporal Lobes. Frontiers in Aging 
Rousset, O. G., Ma, Y., \& Evans, A. C. (1998). Correction for partial volume effects in PET: principle and validation. Journal of Nuclear Medicine $\square$ : Official Publication, Society of Nuclear Medicine, 39(5), 904-11. Retrieved from http://www.ncbi.nlm.nih.gov/pubmed/9591599

Rubin, T. N., Koyejo, O., Gorgolewski, K. J., Jones, M. N., Poldrack, R. A., \& Yarkoni, T. (2017). Decoding brain activity using a large-scale probabilistic functional-anatomical atlas of human cognition. PLOS Computational Biology, 13(10), e1005649. http://doi.org/10.1371/journal.pcbi.1005649

Salat, D. H., Buckner, R. L., Snyder, A. Z., Greve, D. N., Desikan, R. S. R., Busa, E., ... Fischl, B. (2004).

Shafto, M. A., \& Tyler, L. K. (2014). Language in the aging brain: The network dynamics of cognitive decline and preservation. Science, 346(6209), 583-587. http://doi.org/10.1126/science.1254404

Smith, S. M., Vidaurre, D., Beckmann, C. F., Glasser, M. F., Jenkinson, M., Miller, K. L., ... Van Essen, 17(12), 666-682. http://doi.org/10.1016/j.tics.2013.09.016

Spreng, R. N., Wojtowicz, M., \& Grady, C. L. (2010). Reliable differences in brain activity between young and old adults: A quantitative meta-analysis across multiple cognitive domains. Neuroscience \& Biobehavioral Reviews, 34(8), 1178-1194. http://doi.org/10.1016/j.neubiorev.2010.01.009 
Taylor, P. A., Gohel, S., Di, X., Walter, M., \& Biswal, B. B. (2012). Functional covariance networks: obtaining resting-state networks from intersubject variability. Brain Connectivity, 2(4), 203-17. http://doi.org/10.1089/brain.2012.0095

Taylor, W. D., Steffens, D. C., McQuoid, D. R., Payne, M. E., Lee, S.-H., Lai, T.-J., \& Krishnan, K. R. R. (2003). Smaller orbital frontal cortex volumes associated with functional disability in depressed elders. Biological Psychiatry, 53(2), 144-149. http://doi.org/10.1016/S0006-3223(02)01490-7

Tibshirani, R. (1996). Regression Selection and Shrinkage via the Lasso. Journal of the Royal Statistical Society B. http://doi.org/10.2307/2346178

Yuan, R., Di, X., Kim, E. H., Barik, S., Rypma, B., \& Biswal, B. B. (2013). Regional homogeneity of resting-state fMRI contributes to both neurovascular and task activation variations. Magnetic Resonance Imaging, 31(9), 1492-500. http://doi.org/10.1016/j.mri.2013.07.005

Zhang, Z., Liao, W., Xu, Q., Wei, W., Zhou, H. J., Sun, K., ... Lu, G. (2017). Hippocampus-associated causal network of structural covariance measuring structural damage progression in temporal lobe epilepsy. Human Brain Mapping, 38(2), 753-766. http://doi.org/10.1002/hbm.23415

Zhang, Z., Liao, W., Zuo, X.-N., Wang, Z., Yuan, C., Jiao, Q., ... Liu, Y. (2011). Resting-state brain organization revealed by functional covariance networks. PloS One, 6(12), e28817. http://doi.org/10.1371/journal.pone.0028817

Ziegler, G., Penny, W. D., Ridgway, G. R., Ourselin, S., \& Friston, K. J. (2015). Estimating anatomical trajectories with Bayesian mixed-effects modeling. NeuroImage, 121, 51-68. http://doi.org/10.1016/j.neuroimage.2015.06.094

Ziegler, G., Ridgway, G. R., Blakemore, S.-J., Ashburner, J., \& Penny, W. (2017). Multivariate dynamical modelling of structural change during development. NeuroImage, 147, 746-762. http://doi.org/10.1016/j.neuroimage.2016.12.017

Zuendorf, G., Kerrouche, N., Herholz, K., \& Baron, J.-C. (2003). Efficient principal component analysis for multivariate 3D voxel-based mapping of brain functional imaging data sets as applied to FDG- 
bioRxiv preprint doi: https://doi.org/10.1101/490292; this version posted June 25, 2019. The copyright holder for this preprint (which was not certified by peer review) is the author/funder, who has granted bioRxiv a license to display the preprint in perpetuity. It is made available under aCC-BY-NC-ND 4.0 International license. 
Table 1 List of interregional causal influences of metabolic activity identified at $\lambda=0.162$ using LASSO

630 (least absolute shrinkage and selection operator) regression.

\begin{tabular}{|c|c|c|c|c|}
\hline & From & $\rightarrow$ & & To \\
\hline $\begin{array}{c}\mathrm{IC} \\
\#\end{array}$ & Label & $\begin{array}{c}\text { Sig } \\
\mathrm{n}\end{array}$ & $\begin{array}{c}\mathrm{IC} \\
\#\end{array}$ & Label \\
\hline 52 & $\begin{array}{l}\text { Thalamus, brainstem } \\
\text { Supplementary motor area, paracentral }\end{array}$ & - & 27 & Precuneus \\
\hline 44 & $\begin{array}{l}\text { lobule } \\
\text { Insula, inferior frontal gyrus, superior } \\
\text { temporal pole }\end{array}$ & + & 52 & $\begin{array}{l}\text { Thalamus, brainstem } \\
\text { Temporal pole, medial orbitofrontal } \\
\text { cortex }\end{array}$ \\
\hline 63 & Temporal pole & + & 40 & Left middle temporal gyrus \\
\hline 63 & $\begin{array}{l}\text { Temporal pole } \\
\text { Insula, inferior frontal gyrus, superior }\end{array}$ & + & 27 & Precuneus \\
\hline 44 & temporal pole & - & 20 & Right inferior and middle frontal gyrus \\
\hline 63 & $\begin{array}{l}\text { Temporal pole } \\
\text { Insula inferior frontal gyrus sunerior }\end{array}$ & - & 38 & Left thalamus, caudate, lingual gyrus \\
\hline 44 & temporal pole & - & 16 & Superior and middle frontal gyrus \\
\hline 39 & Superior parietal lobule, precuneus & + & 20 & $\begin{array}{l}\text { Right inferior and middle frontal gyrus } \\
\text { Posterior cingulate cortex, precuneus, }\end{array}$ \\
\hline 19 & Basal ganglia, amygdala, insula & - & 46 & lingual gyrus \\
\hline 63 & Temporal pole & - & 19 & Basal ganglia, amygdala, insula \\
\hline 63 & $\begin{array}{l}\text { Temporal pole } \\
\text { Lobule VII crus, Lobule VI hemisphere, }\end{array}$ & - & 34 & Inferior frontal gyrus \\
\hline 81 & vermis & - & 6 & Lobule VII crus \\
\hline 39 & Superior parietal lobule, precuneus & + & 19 & Basal ganglia, amygdala, insula \\
\hline 19 & $\begin{array}{l}\text { Basal ganglia, amygdala, insula } \\
\text { Orbitofrontal cortex, superior temporal }\end{array}$ & - & 17 & Lingual gyrus, calcarine sulcus, cuneus \\
\hline 48 & pole & + & 39 & Superior parietal lobule, precuneus \\
\hline 28 & $\begin{array}{l}\text { Inferior parietal lobule, precuneus } \\
\text { Orbitofrontal cortex, superior temporal }\end{array}$ & + & 17 & Lingual gyrus, calcarine sulcus, cuneus \\
\hline 48 & $\begin{array}{l}\text { pole } \\
\text { Orbitofrontal cortex, superior temporal }\end{array}$ & + & 47 & Postcentral gyrus, inferior parietal lobule \\
\hline 48 & pole & + & 56 & Superior and middle occipital gyrus \\
\hline 28 & Inferior parietal lobule, precuneus & + & 56 & Superior and middle occipital gyrus \\
\hline 28 & Inferior parietal lobule, precuneus & + & 43 & Lobule VII and VIII, hemisphere \\
\hline 48 & $\begin{array}{l}\text { pole } \\
\text { Orbitofrontal cortex, superior temporal }\end{array}$ & + & 11 & Lingual gyrus, calcarine sulcus, cuneus \\
\hline 48 & pole & + & 69 & Inferior occipital and fusiform gyrus \\
\hline 80 & $\begin{array}{l}\text { Inferior parietal lobule } \\
\text { Left inferior frontal gyrus, left middle }\end{array}$ & - & 56 & Superior and middle occipital gyrus \\
\hline 5 & occipital gyrus & - & 28 & Inferior parietal lobule, precuneus \\
\hline 78 & $\begin{array}{l}\text { Postcentral gyrus } \\
\text { Left inferior frontal gyrus, left middle }\end{array}$ & - & 12 & Superior and middle occipital gyrus \\
\hline 5 & occipital gyrus & + & 80 & Inferior parietal lobule \\
\hline
\end{tabular}


bioRxiv preprint doi: https://doi.org/10.1101/490292; this version posted June 25, 2019. The copyright holder for this preprint (which was not certified by peer review) is the author/funder, who has granted bioRxiv a license to display the preprint in perpetuity. It is made available under aCC-BY-NC-ND 4.0 International license.

Left inferior frontal gyrus, left middle 5 occipital gyrus

66 Left inferior temporal gyrus

631

632 